Boise State University

ScholarWorks

Computer Science Faculty Publications and

Presentations

Department of Computer Science

$1-1-2018$

\title{
International and Interdisciplinary Perspectives on Children \& Recommender Systems (KidRec)
}

Jerry Alan Fails

Boise State University

Maria Soledad Pera

Boise State University

Natalia Kucirkova

University College London

Franca Garzotto

Politecnico di Milano 


\section{International and Interdisciplinary Perspectives on Children \& Recommender Systems (KidRec)}

\author{
Jerry Alan Fails \\ Boise State University \\ Boise, ID 83725 USA \\ jerryfails@boisestate.edu \\ Maria Soledad Pera \\ Boise State University \\ Boise, ID 83725 USA \\ solepera@boisestate.edu \\ Natalia Kucirkova \\ University College London \\ London, UK \\ n.kucirkova@ucl.ac.uk \\ Franca Garzotto \\ Politecnico di Milano \\ Milan, Italy \\ franca.garzotto@polimi.it
}

IDC '18, June 19-22, 2018, Trondheim, Norway C) 2018 Copyright is held by the owner/author(s). ACM ISBN 978-1-4503-5152-2/18/06.

https://doi.org/10.1145/3202185.3205866

\begin{abstract}
Resources for children are abundant, but finding suitable and appropriate resources for children in our information-rich society can be challenging. Due to this abundance of information, systems to find and recommend appropriate information for children are needed. Recommender systems (RS) for children have only recently begun to be researched. This area of research brings together researchers in education, child-development, computer scientists, designers, and more who address several issues including those related to education, algorithms, ethics, privacy, security. In this workshop we will: discuss and identify issues related to RS designed for children including challenges and limitations, discuss possible solutions to the identified challenges and plan for future research, and of critical importance work to build a community that explicitly looks at these critical issues. This workshop has a specific theme of educationally-related recommendations, but welcomes other child-oriented recommender system contributions.
\end{abstract}

\section{Author Keywords}

Children, recommender systems, ethics, design, evaluation. 
ACM Classification Keywords - Human-centered computing Human computer interaction (HCI) - Information systems Recommender systems

\section{Introduction}

Children's access to technology has dramatically increased in the past fifteen years, which has engendered a significant change in the way they experience play and learning on and off screen. Due to the overwhelming amount volume of digital content and services now available for children, it is critical to design recommender systems (RS) for (and with) children in mind.

Recommender systems (RS) are software implementations that provide suggestions for items to be of use to a user [13]. These suggestions are intended to facilitate the decision-making process. RS can aid children, as well as parents and educators, in locating materials for learning and play, e.g., books, video contents, or digital games, that are not only of interest to the user, but are also developmentally and educationally appropriate. This in turns calls for new design and evaluation approaches - not only in terms of data and algorithms, but also in terms of UX, rethinking the goals of recommendations when dealing with children, as well as the concept of trust and persuasion for this specific target group, taking into account critical issues such as ethics, privacy, and security.

Following the success and interest of participants in the first KidRec in Como, Italy (in the conjunction with ACM Conference on Recommender Systems - RecSys 2017), we propose to hold a second workshop at the upcoming
2018 IDC Conference. The goals of the workshop are threefold: (1) discuss and identify issues related to RS designed for children including challenges and limitations; (2) discuss possible solutions to the identified challenges and plan for future research; (3) continue to build a community to directly work on these important issues. The specific theme of this edition of KidRec will be on RS in the educational domain for children, but we welcome other research papers and position papers related to the design of recommender technologies for children.

\section{Background}

With the increased content available to children online, new ways to better access that information is needed. Recommender systems (RS) detailed in the literature $[1,3,6]$ - for the most part - have been developed to serve adult users, who can offer explicit feedback, write reviews, or purchase items. While RS for adults have been studied for several decades, RS for children are only recently beginning to be studied and are primarily limited to recommenders in education-related environments $[7,8,9]$.

When focused on young audiences, the role of a RS needs to be reformulated, as it is not sufficient nor appropriate for the RS to identify items that match traditional adult users' preferences and interests.

Instead, it is imperative that the recommenders also explicitly consider children's educational and

developmental needs and motivational strategies to engage with content. RS can recommend both multimedia and traditional physical content: for example, children's book finder systems can recommend both printed books and apps. Current RS adopt a business-driven model in which previous 
engagement dictates future recommendation, based on a like-like model. This, however, is not aligned with educational or developmental models of learning. Adultoriented models make several assumptions about the user that are not applicable to the young child, such as for example informed consent or established preferences.

A reflection on these complex issues leads to several questions that need to be answered from an interdisciplinary perspective to allow for a holistic understanding of their impact on individual children. Given that children's access to online content is global, it also requires an international perspective that would incorporate best practice on the use of children's data for educational and commercial purposes from a global viewpoint. Current research lacks behind the reality of many software developments in this area and use of recommender systems in formal and informal learning environments. In light of the urgency of issues such as potential misuse of children's personal data, and the scale of the use of recommender systems in education worldwide, we feel it is necessary to bring an international, multidisciplinary community together to collectively map out the field of RS for children, and to begin to address these issues together.

Goals

As an interactive workshop, KidRec aims to congregate researchers and experts from multiple disciplines, in order to understand the ethical, pedagogical, and technical implications of designing and developing RS for children and educational activities. We intend to do this by accomplishing the following three main goals:
1. Discuss and identify the benefits and limitations related to RS designed for young children;

2. Discuss possible design and research solutions to the identified challenges;

3. Expand the community of professionals interested in RS and children's education.

To the best of our knowledge, there have been no workshops on Children \& Recommender Systems beyond the KidRec we organized at RecSys 2017. Insights from KidRec 2017 reinforced our sense that there is a need for interdisciplinary communities to come together and jointly discuss these challenges.

Furthermore, there is paucity of data that can be used to inform the design and assess recommender systems for children. A number of workshops have been held in the past few years in closely-related/complementary areas, which provide evidence of the interest of the research community in this topic. EdRecSys [5], attracts researchers focused on recommender systems tailored to education. Search as Learning (SAL) [11], held last year at ACM SIGIR, assembled researchers interested in information retrieval, natural language processing, and education. Neither of the aforementioned workshops focus on $\mathrm{K}-12$ populations.

The ACM-sponsored conference Interaction Design and Children (IDC) offers a number of workshops that put children at the forefront $[2,11]$. Following the success and interest of participants in the first KidRec [12], we propose to add to the diversity of workshops offered with children's technology in mind. IDC is a venue that has historically focused on technology development for and with children. Because diversity is welcomed at IDC, we feel it is an ideal venue to continue the 
discussion on growing needs and issues related to child-centered RS

\section{Organizers}

Jerry Alan Fails ${ }^{1}$ (jerryfails@boisestate.edu) is an

Associate Professor in the Computer Science

Department at Boise State University. Jerry's research

is in the area of human-computer interaction, with particular focus on designing, developing, and evaluating technologies with and for children. Jerry has participated on and led participatory design groups where children and adults work together as design partners for the last fourteen years. He has developed and evaluated several technologies for children, most of which are for informal educational purposes and leverage technology to bring children together and encourage them to explore their environment. He has organized workshops and courses at $\mathrm{CHI}$, and reviewed for and served on the program committee for $\mathrm{CHI}$, IDC (Interaction Design and Children) and other conferences and journals.

Natalia Kucirkova (n.kucirkova@ucl.ac.uk) is a Senior Research Fellow at the Department for Learning \& Leadership at University College London, Institute of Education, where she leads the ESRC-funded project Children's Personalised Stories. Her research concerns innovative ways of supporting children's book reading, digital literacy and exploring the role of personalisation in early years. She co-developed an award-winning children's app 'Our Story' and has widely published on early literacy and children's technology. Her research takes place collaboratively across academia, commercial and third sectors. Natalia is a Fellow of the

\footnotetext{
Primary contact person
}

Royal Society for Arts, Chair of the judging panel for the UKLA Children's Digital Book Awards and Advisory Board Member for Save The Children. She co-edits the Bloomsbury Academic Book Series Children's Reading \& Writing on Screen.

Maria Soledad Pera (solepera@boisestate.edu) is an Assistant Professor in the Computer Science Department at Boise State University. Sole's research work focuses on the application of information retrieval information extraction, and natural language processing techniques for developing RS, primarily for children, including book recommenders for $\mathrm{K}-12$ readers and recommenders that target parents \& teachers. Sole's work related to information retrieval applications tailored towards children, such as query suggestion and search intent tools that can enhance the location of educational materials in the Web search environment, has been funded ${ }^{2}$ by the NSF (National Science Foundation). She has served as PC and reviewer for conferences and journals related to RS and Information Retrieval, including ACM RecSys, Argentine Symposium on Artificial Intelligence, and Knowledge and Information Systems journal. She was also one of the co-organizers of International Workshop on Educational Recommender Systems, held in conjunction with 2016 IEEE/WIC/ACM International Conference on Web Intelligence and International Workshop in Children and Recommender Systems held in conjunction with the 2017 ACM RecSys Conference on Recommender Systems. She is also the General Chair of ACM RecSys 2018.

\footnotetext{
2 https://www.nsf.gov/awardsearch/showAward?AWD_ID =1565937
} 
Franca Garzotto (franca.garzotto@polimi.it) is an Associate Professor in Computer Engineering at the Politecnico di Milano - Dept of Electronics, Information, and Bioengineering, where she is responsible for the I3 (Innovative Interactive Interfaces) Lab. Her research interest and activities focus on innovative interfaces and interaction paradigms for children and for persons with special needs. She is an active member of the IDC (Interaction Design and Children) community and has served as Program Chair of 2 editions of the ACM IDC conference. She has contributed to the RS community with research on user-center evaluation of RS, design patterns for RS, and video recommendations. She served as European chair of ACM SIG-WEB (ACM Special Interest Group on Hypermedia \& Web) and she is Member of the Evaluation Panel of ERC (European Research Council) Grants. She has organized over 50 tutorials and workshops, is the recipient of 2 IBM Faculty Awards, and has been project coordinator of 5 large EC funded projects.

\section{Website}

The website will offer potential attendees information pertaining to objectives for the workshop, contact information of organizers, important dates associated with KidRec, information related to work submission, list of accepted papers, and outline of the program.

URL: https://kidrec.github.io/

\section{Pre-Workshop Plans}

We anticipate a call for papers and position papers focusing on open challenges in promising research directions as well as speculative or innovative work in progress. We will reach out to experts in areas related to the topic of our workshop and invite them to submit position papers, which will initiate the conversation concerning the challenges, limitations, and diverse perspectives that prevent the RS' design and development for children.

The workshop will be promoted at conferences including $\mathrm{CHI}$ and also online through various channels, including social media (e.g., Facebook, Twitter) and sending CFP to forums like DBWorld and WikiCFP and relevant mailing lists, such as SIG-IRList, SIG-CHIList, Dev-Europe, ID-Research-UK and IDC email lists.

\section{Workshop Structure}

Overview

We envision KidRec as a full-day, interactive workshop, for between 20 to 30 participants. We anticipate a regular call for short papers (4 pages) discussing novel work in progress and position papers ( 2 page extended abstract) focusing on open challenges in promising research directions as well as speculative or innovative work in progress.

We will select accepted papers through peer-review, for which we will recruit a Program Committee comprised of experts in diverse fields: recommender systems, human-computer interaction, child-computer interaction, information retrieval, educational recommenders, educational professionals, ethics experts, and educational psychologists.

\section{Proposed Activities}

We aim to facilitate a highly participatory [4] workshop in which attendees can discuss the limitations and challenges of RS for children and identify possible solutions and avenues of research. We propose to accomplish this through an interactive format, 


\section{Workshop Schedule}

(Morning Activities)

9:00-9:15 Welcome and introduction by organizers

9:15-9:45 Icebreaker activity

9:45-10:30 Interactive Panel

10:30-11:00 Coffee break

11:00-11:45 Interactive Panel

\section{1:45-12:30 Keynote}

12:30-14:00 Lunch organized in groups for discussions

\section{Interactive Panel}

- Grouped submissions

- 5-8 Ignite presentations

- Table discussion

- Full group discussion, Q\&A

- Everyone writes research questions on sticky notes throughout including: community building exercises, informal interactions, facilitated group work, short paper and position paper presentation. An outline of the activities we envision for the workshop is presented below:

\section{MORNING:}

- 9:00-9:15: Welcome and introduction which will include insights and feedback from KidRec 2017

- 9:15-9:45: Icebreaker activity

Introduce yourself to someone you don't know, talk about your research, recommend 1-2 people they should talk to at RecSys and 1-2 papers they should read that relate to their work. Switch. (So everyone is introduced to new people and has referrals to 2-4 other people.)

- 9:45-10:30: Interactive Panel 1 (See panel description below

- 10:30-11:00: Morning Coffee Break

- 11:00-11:45: Interactive Panel 2

- 11:45-12:30: Keynote

- 12:30-14:00: Facilitate lunch group organization, issue lunch discussion assignment

LUNCH: $12: 30-14: 00$

\section{AFTERNOON:}

- 14:00-14:30: Report on lunch discussion assignment

- 14:30-15:15: Separate points of view $\rightarrow$ identify problems

Sticky note frequency analysis - make small groups, but each individual writes down ideas for problems (one per sticky note), can discuss amongst groups as they write, workshop facilitators group and perform an informal frequency analysis of sticky notes, and have a brief the whole group about the identified problem areas and discuss groups identified, missed, etc.

- 15:15-15:45: Afternoon Coffee Break

- 15:45-16:00: Debrief problem identification

- 16:00-17:00: Comprehensive point of view $\rightarrow$ possible solutions

- Low-tech/paper prototypes - create new small groups, and have the group focus on one of the problems identified and design a solution together.

- Share designs with group.

- 17:00-17:30: Agreement on directions of the future work (outline future research in the area)

INTERACTIVE PANEL SESSION FORMAT

- Submissions will be organized and grouped to create different interactive panel sessions

- 15 minutes: Ignite!-style presentations of 5-8 workshop participants

- 10 minutes: table discussions about the panel round tables will be requested and each table will have 3-6 participants to have a small group discussion about the items discussed

- 20 minutes: Full group questions and answers, and whole group discussion

- We could utilize a technology back-channel such as todaysmeet or padlet.

\section{KEYNOTE}

In keeping with our proposed the theme of educationally-oriented recommender systems for this the second KidRec workshop, upon acceptance of the workshop we will identify a suitable keynote speaker and extend an invitation so that she/he can offer an overview of the needs for RS in the educational domain for children. 


Workshop Schedule
(Afternoon Activities)
14:00-14:30 Reports on lunch
discussion
14:30-15:15 Map research
questions
15:15-15:45 Coffee break
15:45-16:00 Debrief problem
identification
16:00-17:00 Merge maps,
identify what has been done,
what is being done, what to do
17:00-17:30 Wrap-up and
discuss next steps

Resources Needed for Workshop

- To facilitate ice breakers and discussion activities, we anticipate round-table organization, as opposed to classroom setting.

- Due to paper presentations, screen and projector would be ideal.

- To engage attendees on sticky-note problemsolving activities, we will need a whiteboard and/or a few poster frames.

\section{Post-Workshop Plans}

Accepted short papers and position papers will be published on http://ceur-ws.org/ and on our workshop website. A report on discussion and findings from workshop interactions will be submitted to venues like SIGIR Forum, IDC, SIGCHI. We will also seek the opportunity for a special issue on KidRec in a journal such as User Modeling and User-Adapted Interaction Journal (UMUAI) Journal (Springer), ACM Transactions on Interactive Intelligent Systems (TiiS), or The International Journal of Child-Computer Interaction (Elsevier), ACM Transactions on Computer Human Interaction (Tochi), International Journal of HumanComputer Studies, Interacting with Computers, Behaviour \& Information Technology.

Based on attendees' interests, we anticipate creating a mailing list to facilitate continuing dialogue among researchers interested in RS for children.

\section{Call for Participation}

With increasingly large amounts of educational content online, recommender systems (RS) for children are becoming particularly important. When focused on this young audience, it is not sufficient for recommenders to identify items that match children's preferences and interests. Instead, it is imperative that they also consider children's needs from multiple perspectives: educational, developmental, and motivational, to name a few. Considering these particular needs opens a rich set of questions to answer:

- What are the benefits of child-specific recommendations?

- What role do age, developmental stage, socioeconomic status, curricular standards play in RS for children?

- What are the goals of RS for children: persuade, educate, guide, and support their learning, motivation to learn or something else entirely?

- What are the ethical and privacy challenges associated with RS for children?

The goal of this interactive, full-day workshop is to share and discuss research and projects that reach beyond classic recommender techniques. We invite submissions related to recommender systems for children including papers concerned with: educational technology for children; recommender algorithms for children; recommendation explanations; privacy of children's data; transparency; expert-in-the-Loop (e.g., teacher, parent); child-centered content domains (e.g., leisure, education); user profiling; personalization; gamification; mediation, to name a few.

We invite short papers (4-6 pages) discussing nove work in progress and position papers (2-4 pages) focusing on open challenges in promising research directions as well as speculative or innovative work in progress. All papers will be peer-reviewed, and at the time of submission, must not be under review in any other conference, workshop or journal. 
At least one author of each accepted paper must register for and attend the workshop and main conference.

For further information, see our website:

https://kidrec.github.io/

\section{References}

1. Adomavicius, G., \& Tuzhilin, A. (2005). Toward the next generation of recommender systems: $A$ survey of the state-of-the-art and possible extensions. IEEE transactions on knowledge and data engineering, 17(6), 734-749.

2. Designing Tangibles for Children. http://www.antle.iat.sfu.ca/IDC2016TangiblesChild renWorkshop/index.html

3. Ekstrand, M. D., Riedl, J. T., \& Konstan, J. A. (2011). Collaborative filtering recommender systems. Foundations and Trends in HumanComputer Interaction, 4(2), 81-173.

4. Faciliating participatory workshops https://we.riseup.net/assets/25682/FacilitatingWor kshops.pdf

5. International Workshop on Educational Recommender Systems (EdRecSys). https://edrecsys.wordpress.com/

6. Lops, P., De Gemmis, M., \& Semeraro, G. (2011). Content-based recommender systems: State of the art and trends. In Recommender systems handbook (pp. 73-105). Springer US.

7. Manouselis, N., Drachsler, H., Vuorikari, R., Hummel, H., \& Koper, R. (2011). Recommender systems in technology enhanced learning. In Recommender systems handbook (pp. 387-415). Springer US.

8. Pera, M. S., \& Ng, Y. K. (2014, October). Automating readers' advisory to make book recommendations for $\mathrm{k}-12$ readers. In Proceedings of the 8th ACM Conference on Recommender systems (pp. 9-16). ACM.

9. Kucirkova, N., \& Cremin, T. (2017). Personalised reading for pleasure with digital libraries: towards a pedagogy of practice and design. Cambridge Journal of Education, 1-19.

10. Philosophy with Children: Helping Designers Cooperate with Children.

http://www.industrialdesigncenter.be/idc2016/

11. Search as Learning Workshop. http://chauff.github.io/sal2016/

12. Jerry Alan Fails, Maria Soledad Pera, Franca Garzotto, and Mirko Gelsomini. 2017. KidRec: Children \& Recommender Systems: Workshop Colocated with ACM Conference on Recommender Systems (RecSys 2017). In Proceedings of the Eleventh ACM Conference on Recommender Systems(RecSys '17). ACM, New York, NY, USA, 376-377.

13. Ricci, F., Rokach, L. and Shapira, B. eds., 2015. Recommender systems handbook. Berlin, Germany: Springer. 